STRUCTURAL SCIENCE CRYSTAL ENGINEERING MATERIALS

ISSN 2052-5206

\section{Chemistry of Carbon Nanostructures. Edited by Klaus Müllen and Xinliang Feng. De Gruyter, 2017. Hardcover, Pp. XI+319. Price EUR 89.95, USD 126.00, GBP 67.99. ISBN 978-3-11-028450-8.}

\author{
Alain Celzard* and Vanessa Fierro* \\ Université de Lorraine, CNRS, IJL, Epinal, F-8800, France. *Correspondence e-mail: alain.celzard@univ-lorraine.fr, \\ vanessa.fierro@univ-lorraine.fr
}

Keywords: book review; carbon nanostructures.

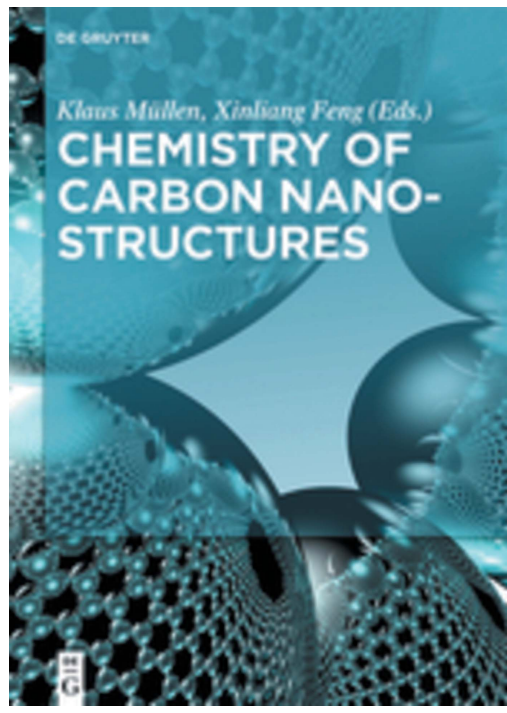

Chemistry of Carbon Nanostructures is divided into ten chapters and presents various contributions to the quite broad topic of carbon nanomaterials and $\pi$-conjugated molecules. Given the major importance of nanocarbons at the present time, especially in view of the rapid expansion of research devoted to graphene and related materials, this book is timely and most welcome.

Whereas the quality of editing and printing is obvious, it is unfortunate that, as it is already the case for many other books, there is no linking of ideas between the chapters. Neither a foreword nor an introduction is present for explaining the content, the rationale behind the order of the chapters or the reason for having selected the themes that have been addressed. Indeed, much more might have been expected from the very broad and general title of the book, whereas one finds that very specialized knowledge is reported in the different chapters. One might add that, when considering the topic of carbon nanostructures, it would have been rather easy to order the chapters and to provide a more self-consistent book, for instance gathering themes related to graphene on the one hand, themes related to nanorings, nanocages or nanoribbons on the other hand, etc. Instead of this, totally unrelated chapters follow one other.

The title of chapter 1 is Carbon Nanomembranes (29 pages), but only membranes produced by electron-induced cross-linking of aromatic self-assembled monolayers are reviewed. Due to this, the specific case of polyaromatic thiols is considered in detail. The review is very complete and goes deep into the formation mechanisms of these materials and in the way those mechanisms were elucidated. Examples of materials prepared in various conditions are presented, showing the versatility of such membranes, as well as the various techniques used for investigating them. At the end, a few properties and potential applications are commented on. Very interesting, but quite specific. The chapter lacks subsections that would have improved the reader's experience and increased the clarity on the whole.

Chapter 2 (37 pages), entitled Controlled Functionalization of Graphene by Oxoaddends deals with a special family of graphene oxide, called 'oxo-functionalized graphene', corresponding to graphenic domains having an intact carbon framework. Compared with the other chapters of this book, this one received less care at the moment of being written, as proved by various typos, flawed English here and there, and incorrect section numbering listed in the introduction. A significant part of the chapter is devoted to the preparation of oxo-functionalized graphenes by various routes and to their structure and characterization tools. Chemical functionalization and modification is then considered. In this part, several figures lack clarity. However, this chapter is of great importance considering there is worldwide research interest for graphene and its relatives, especially for those prepared by simple methods in solutions, and for all related problems of defects, polydispersity and stability.

In chapter 3 (Chemical Synthesis of Cycloparaphenylenes, 37 pages), fine organic chemistry is used for preparing carbon nanorings that might be considered as templates or building blocks for the construction of carbon nanotubes. A thorough review detailing how such nanorings can be synthesized, whether armchair, zigzag or chiral, doped or not with heteroelements, is extensively given, and constitutes the major part of the chapter. 
The latter is not always easy to follow because of the very specific and dense terminology of organic chemistry, and the reference to quite a lot of molecules by numbers rather than their chemical names all through the chapter. The last part is dedicated to the synthesis of carbon nanocages, but how all these structures might be combined and used in materials science is not explained, nor are perspectives given.

Chapter 4 (Controlled Chemical Synthesis in CVD Graphene, 42 pages) reviews the conditions for growing monolayers, bilayer or few-layer graphene on various substrates. One may bemoan the significant use of too vague generalities, especially with the excessive use of words such as «high» or «low», and the corresponding lack of explicit data. The multi-parameter nature and the intrinsic complexity of the CVD processes may explain it. Many figures are not always very clear and their captions are for the most part insufficient for understanding completely what is being shown. The English should also have advantageously been polished prior to publication. A large table of experimental conditions and references finally saves the day. Just as for chapter 2 , this chapter is among the most important in the book for graphene scientists as it reports the problems and the successes of a very famous method for getting large and almost flawless graphene sheets. The extensive bibliography is another highlight of this section.

The fifth chapter (27 pages) deals with Chemical Functionalization of Graphene Family Members. Very important reminders about graphene, graphene oxide and reduced graphene oxide are given, and key reactions for modifying their surface chemistry are reviewed. Thus, non-covalent and covalent functionalization reactions are explained, and a lot of very useful details are provided as for the latter. Next, a brief overview of usual physicochemical techniques for characterizing the raw and modified materials is given. Since the major part of the book deals with graphene and its relatives, this chapter should have been the first of the series of ten: not only does it introduce the topic quite well, but the reactions and the techniques are also used in the other parts of the book. Also noteworthy is that the content of this chapter will be useful to any carbon scientist, i.e. not only those working on carbon-based nanomaterials. To be read first and foremost.

Graphene via Molecule-Assisted Ultrasound-Induced Liquid-Phase Exfoliation: A Supramolecular Approach is examined in chapter 6 (21 pages), the only one of the book dedicated to a top-down approach. It explains what exfoliation of graphite induced by ultrasounds is, and why the assistance of more or less polar molecules is helpful, depending on the solvent in which the experiments are carried out. Although the molecular interactions are suitably explained, accounting for the degree of separation of graphene layers and for the final concentration of the resultant suspensions, one may regret the lack of experimental details; for instance details about optimal power and duration of sonication, and about optimal amounts of graphite and solvents to be handled, etc. This is another important chapter for people interested in large-scale and lowcost production of graphene.
In chapter 7, the bottom-up Solution Synthesis of Atomically Precise Graphene Nanoribbons (32 pages) is addressed. After a definition of what graphene nanoribbons are, what their expected properties are, and why they are of great interest in various kinds of electronic devices, their possible structures are then discussed. The main chemical reactions, precursors and preparation steps required for synthesizing them are then reviewed, and the corresponding advantages and drawbacks of each are given. Chemical modifications are next introduced with emphasis on nitrogen doping, as the latter allows tuning the semiconducting properties. Finally, the most relevant characterization methods are reviewed with examples, demonstrating their importance for concluding whether the appropriate nanoribbon structure has been obtained after synthesis. The chapter ends with the main challenges to be faced and the first solutions, giving also perspectives and ideas for future work. Very interesting and well explained.

Chapter 8 (Nanodiamonds for Biological Applications, 27 pages) is the only one dealing with (almost) purely $s p^{3}$ nanocarbon. It reviews the amazing properties and successful/ potential uses of those nanomaterials which have been given less exposure to the general public, but which deserve the greatest interest due to their outstandingly broad range of high-technology applications. This part of the book provides many examples proving the biocompatibility of nanodiamonds, both in vitro and in vivo, and reviews the chemical modification methods for enhancing further their stability and compatibility with biological environments. Their functionalization is also considered for applying them to drug delivery, sensing and imaging. Besides, it is explained how their special magnetic properties can be used for nanoscale magnetometry, thermometry and single-molecule detection, amongst others. The chapter closes with further progress that still needs to be done. Impressive. The only regret here is the lack of information about the preparation of such amazing nanomaterials and how they should be handled.

The ninth chapter, Polycyclic Hydrocarbons with an OpenShell Ground State (36 pages), reports on the very rich chemistry of such kinds of organic, $\pi$-conjugated molecules and on their many possible precursors, combinations and derivatives, and properties. The major part of the chapter requires from the reader a high level of background knowledge of theoretical chemistry and organic chemistry, and this is the only criticism to be made here: almost no reminder is given, no effort is made to familiarize the reader with the concepts, and the authors go straight into their highly specific topic. When one is not used to the concepts reported here, this chapter is probably the most difficult to read as the content is presented at quite a high academic level. However, there is a lot to learn here, and the possible effort that is required is worth it when considering the multiple technological applications that these organic molecules may have.

The last chapter 'Synthesis and Use of Reactive Molecular Precursors for the Preparation of Carbon Nanomaterials' (25 pages) stands out by its clarity. It deals with the synthesis and the reactivity of polyynes, or, more exactly oligoynes due to 
their limited molecular sizes, for preparing carbon nanostructures in amazingly mild conditions, i.e. slightly above room temperature. This chapter acknowledges the difficulty of identifying positively the new carbon nanostructures that may form from such precursors, but the reader will promptly be convinced of the significance of such a topic of growing interest. One might have welcomed a short section about theoretical predictions that have been made on $s p$-hybridized carbons, sometimes making them be called the next miracle material (after fullerenes, nanotubes and now graphene also received the same qualifying term).

In conclusion, we here deal with an eclectic book based on a series of chapters of uneven quality, not linked to one other and sometimes lacking conclusions and perspectives, but all correctly adhering to the general term of 'carbon nanostructures'. Surprisingly, no chapter mentions or even considers the chemistry of fullerenes and carbon nanotubes, but this is probably due to the fact that those topics have already been extensively addressed in a number of earlier monographs. However, their complete absence makes the title of the book somewhat misleading because one may expect that these topics are also considered, but are actually not.
Whereas several chapters address similar questions by different means, no cross references are made that would have helped the reader; for instance, for comparing materials and methods. Moreover, some very similar reminders about graphene in the introductions of several different chapters might have been avoided. The book must thus be considered as a collection of independent, sometimes highly specific, reviews. Due to this, it is unlikely that readers will be equally interested in all chapters. However, this criticism applies to many books nowadays, and from this point of view, the work edited by Müllen and Feng is certainly not worse than others. In contrast, the style is self-consistent, the illustrations are most of the time of very high quality, and the text is very clear and helpful. Most importantly, this is a book in which anyone will find something to learn, whether physicist, chemist, nanotechnologist, etc. What the title does not explicitly explain, but what the chapters' contents show, is that this book mainly deals with graphene and its relatives. Any researcher deeply involved in the 'graphene' topic should, therefore, have it on his/her shelf. It thus appears that the present book is a musthave in the library of any specialist in nanotechnology and/or carbon scientist. 